\title{
Investigation of Small Motions of Liquid in Cylindrical Chamber of Auto-Balancing Device
}

\author{
Ilona DRACH*, Algimantas BUBULIS**, Darius MAŽEIKA***, \\ Rasa KANDROTAITÉ JANUTIENE $* * * *$, Darius JUODVALKIS***** \\ *Khmelnytsky National University, Institutskaya 11, Khmelnitskiy, 280016, Ukraine, E-mail: cogitare410@gmail.com \\ **Kaunas University of Technology, Mechatronics Institute, Studentu 56, LT-51424, Kaunas, Lithuania, \\ E-mail: algimantas.bubulis@ktu.lt \\ ***Kaunas University of Technology, Faculty of Mechanical Engineering and Design, Studentu 56, LT-51424, Kaunas, \\ Lithuania, E-mail: darius.mazeika@ktu.lt \\ ****Kaunas University of Technology, Faculty of Mechanical Engineering and Design, Studentu 56, LT-51424, Kaunas, \\ Lithuania, E-mail: raskand@ktu.lt \\ *****Kaunas University of Technology, Faculty of Mechanical Engineering and Design, Studentu 56, LT-51424, Kaunas, \\ Lithuania, E-mail: darius.juodvalkis@ktu.lt
}

cross $^{\text {ref }}$ http://dx.doi.org/10.5755/j01.mech.24.2.20402

\section{Introduction}

For machines with variable rotor imbalance and when balancing rotors of machines without stopping under operating conditions, traditional methods of reducing vibrations are ineffective. These machines include separators and centrifuges that are used in various sectors of the national economy (food, chemical, sugar, mining, etc.), medicine, and everyday life. The most reliable, promising, and often the only possible method for reducing the vibrations of such machines is automatic balancing with the help of devices with the free movement of correction masses, which have the form of a hollow chamber partially filled with working bodies (liquid), and are passive direct action regulators that do not require power supply and control system for moving correction masses [1].

The idea fluid balancer is thus not new; but recently there has been a renewed interest, both in industry and in academia [2]. Experimental fluid balancer studies have been carried out by Kasahara [3] and Nakamura [4]. As to mathematical models, simple lumped mass models have been considered by Bae [5], Jung [6], Majewski [7], Chen [8], and Urbiola-Soto and Lopez-Parra [9]. The first and the last two of these papers include experimental studies a swell. The paper [6] includes a few numerical simulation results based on computational fluid dynamics.

Making a fluid balancer to work perfectly is a delicate process and, thus, a more detailed understanding of the fluid dynamics of the balancer is desirable. This is the main motivation behind the present work.

The characteristic features of the process of automatic balancing of liquid under stationary conditions of rotor movement with a vertical axis of rotation is that automatic fluid balancing is effective for elastically deformable rotors, on elastic supports ones, where there is a phase difference between the direction of force from the imbalance and the rotor deflection or the displacement of the rotor, as well as the fact that in the auto-balancer device chamber the liquid tends to set against an imbalance not only in the above resonance but also in the preresonant area of rotation of the rotor and at the resonance itself [10]. However, there is no theoretical verification of fluid behavior in the auto-balancing device (ABD) cham- ber under non-stationary conditions of rotor rotation.

Under some simplified assumptions it is [11] shown that the risk of loss of stability of a system containing a cavity partially filled with a liquid always exists where the intrinsic frequency of any form of free fluid fluctuations is close to the nutation frequency of the carrier fluid of the body. An infinite number of forms of free fluctuation of a fluid corresponds to an infinite number of areas of instability, however, as shown in [12], only a few first regions can have practical value. It is believed that the internal friction that damps its own fluctuations in the fluid reduces the region of higher order instability.

The purpose of the paper is to analyze the behavior of a fluid in an auto-balancing device chamber for rotors of machines with a vertical axis of rotation in the case of a non-stationary movement of the shaft-fluid ABD system, where it is necessary to take into account the fluctuations of the free surface of the liquid.

\section{Experimental details}

In [14] it is shown that the free surface of a fluid is part of a paraboloid of rotation with an axis that coincides with the main central axis of the inertia of the system. As the velocity increases, the parabolic form gets into a cylindrical one. In the horizontal section of the ABD chamber, the free surface of the fluid is a circle. Reducing the radius of the free surface and correspondingly increasing the thickness of the liquid layer at a constant radius of the chamber $R$ does not cause a shift in the centre of the mass of the system, since the centre of the free surface of the liquid coincides with the centre of the mass of the system. In the change of the unbalanced state of the rotary system, a thin layer of fluid is involved, which is close to the magnitude of the double displacement of the centre of the mass of the rotor $[10,13]$. Another liquid only increases the mass of the system, located concentrically around the axis of rotation. However, it has been experimentally determined that the amplitude of the system fluctuations increased at a critical frequency range when the ABD chamber was filled with more fluid than necessary for balancing.

To investigate fluid behavior in an auto balancing 
device in the pre-resonance and above resonance areas, an ABD model of optically transparent material was developed and made. The ABD is a ring with an outer diameter $\varnothing 400 \mathrm{~mm}$, two concentric partitions $\varnothing 300$ and $\varnothing 200 \mathrm{~mm}$ in diameter, forming three concentric chambers for the arrangement of working bodies in them. The installation for studying the behavior of working bodies in the ABD is a hard console vertical rotor 1 elastically suspended to the body 5 (Fig. 1). To ensure sufficient rotor rigidity and the ABD 7 fixed on it, the first is shaped like a hollow cylinder of sheet stainless steel with a bottom at one end, which is rigidly secured to the shaft. The axis of the rotor through the rolling bearings 3 is connected to the rigid platform 2 connected to the body of the unit 5 by four elastically damping pendants 4 (the actual frequency of oscillations of the suspension system is $\sim 9-10 \mathrm{~Hz}$ ).

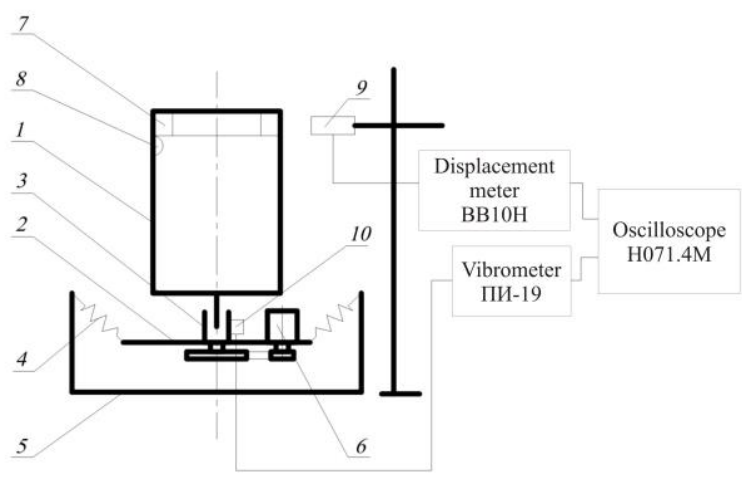

Fig. 1 Installation for studying the behavior of working bodies in the ABD: 1 - drum (rotor); 2 - platform; 3 - bearing housing; 4 - elastic elements; 5 - body; 6 - electric motor; 7 - ABD; 8 - imbalance; 9 - inductive sensor; 10 - piezo sensor (accelerometer) scheme
At the free end of the hollow cylinder (further on the rotor), a model of ABD 7 is inserted, which is inserted into the rotor and fixed to it by the outer diameter with the help of clamps. Due to this, the necessary stiffness of fastening of the $\mathrm{ABD}$ on the rotor and co-ordination are provided. The ABD model has an inner opening sufficient for free access to the inner cavity of the rotor, which allows, without dismantling the ABD, to change the rotor's imbalance 8 both in location and size by attaching it to the inner wall of the specified load, which during the research additionally holds the centrifugal by forces. The rotor drive is carried out by a synchronous electric motor 6 with a power output of 180 watts through a lowering clutch transmission. The motor is powered by alternating current, whose voltage can vary in a wide range with the help of an autotransformer with a control system. Thanks to this, the installation allows you to set the rotation of the rotor with different frequencies in the range of $0.5-18 \mathrm{~Hz}$ (30$1080 \mathrm{rpm}$ ), to set different angular accelerations when accelerating the rotor, to stop in the pre-resonance and above resonance areas of rotation of the rotor on a given time for detailed research on the work of the ABD.

In order to determine the effect of the volume of liquid on the efficiency of the system balancing on the resonant rotor frequencies, a series of studies with an imbalance $D=3000 \mathrm{~g} \cdot \mathrm{cm}$ for a variety of radii of the chambers ABD $(R=0.2,0.15,0.1 \mathrm{~m})$ separately where performed. The chamber stepwise (in $50 \mathrm{ml}$ increments) was filled with liquid (fresh water). After each change in the volume of the liquid, the vibration of the upper edge of the rotor was recorded during its movement. The results of processing records are presented on Fig. 2.

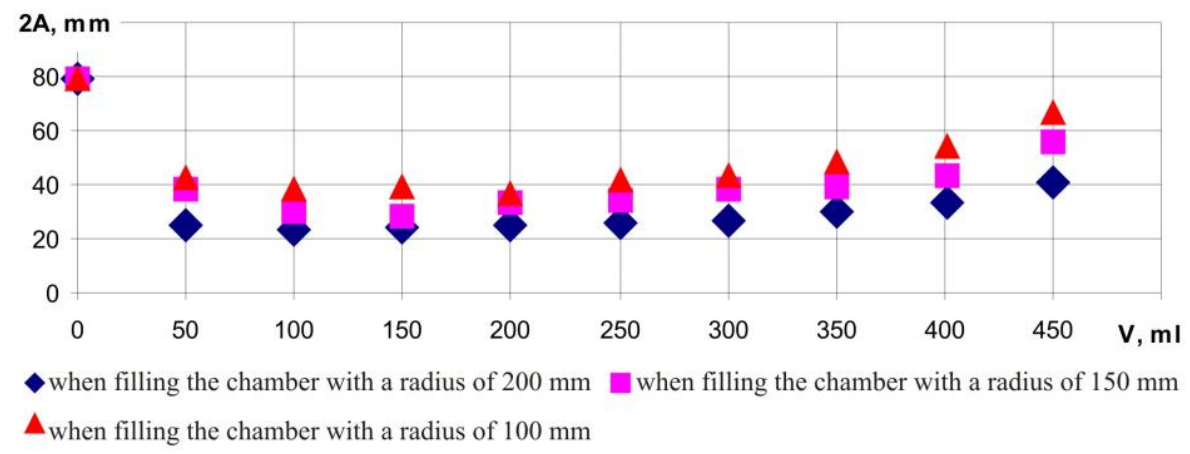

Fig. 2 Amplitude of fluctuations of the upper edge of the drum at different volumes of the working fluid in the ABD

From Fig. 2 it is seen that automatic balancing with liquid working bodies for a vertical rotor at the resonance itself is obvious (comparing with the points corresponding to $V=0 \mathrm{ml}$ ). There is an optimal amount of fluid for efficient balancing of the rotor, it is $100 \mathrm{ml}$ for a radius of $200 \mathrm{~mm}, 150 \mathrm{ml}$ for a radius of $150 \mathrm{~mm}$ and $200 \mathrm{ml}$ for a camera with a radius of $100 \mathrm{~mm}$ with a mass of imbalance of $150 \mathrm{~g}$. In cases where the volume of the working fluid increases, there is an increase in the amplitudes of oscillations.

To explain this behaviour of the system we consider the conditions of wave formation on the free surface of the liquid. To do this, we will analyze the frequency equation of the system of differential equations, which describe the motion of a rotating rotor with an $\mathrm{ABD}$ and a liquid inside it.

\section{Results and discussion}

We accept the calculation scheme, shown in Fig. 3. Let us suppose that a simplified flat task is considered (since the geometric dimensions of the ABD have a ratio $R>>h$ [10]); that the liquid involves into the cylinder chamber of the ABD and rotates with the rotor as a single solid, and that, when neglected by gravitational forces, it has the form of a ring with an internal radius $R_{1}$ and external $-R$. We shall consider the small translational motion of the cylinder in the plane of rotation $(z=z(t)$ and $y=y(t)$ are the generalized coordinates of the cylinder axis). In this case, the movement of the liquid in relation to the cylinder 


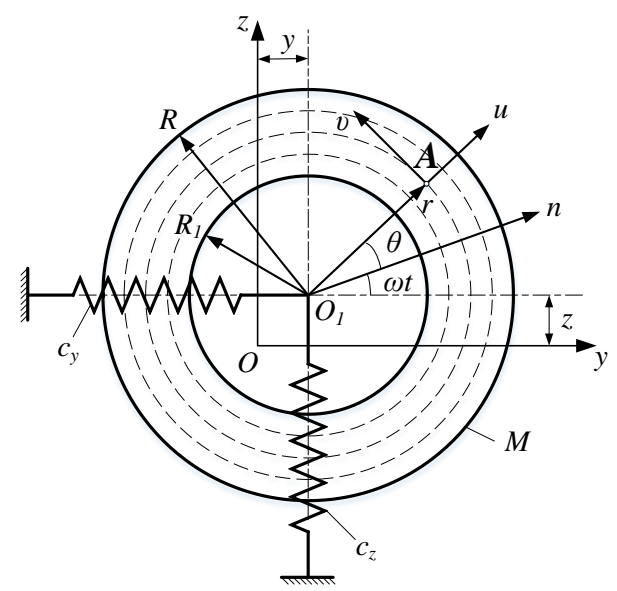

is also considered as small one. In a relative motion, viscosity strengths are not taken into account.

For a moving coordinate system connected with a rotating cylinder, we take the polar coordinate system $O_{1}$ with the polar axis $n$ (Fig. 3); $r, \theta$ - coordinates of A point in this system. In addition, in Fig. 3 the following notation is introduced: $u=u(r, \theta, t) ; v=v(r, \theta, t)$ - respectively, the radial and tangential components of the relative velocity of the liquid at the point $(r, \theta)$. Denote $M$ - mass of rotor with ABD.

Free oscillations of the rotor-fluid system are described linearized (due to the smallness of the considered motions of the system) by differential equations in the chosen polar coordinate system:

Fig. 3 Calculation scheme

$$
\frac{\partial u}{\partial t}-2 \omega v=-\frac{1}{\rho} \frac{\partial p}{\partial r}-\ddot{y} \cos (\omega t+\theta)-\ddot{z} \sin (\omega t+\theta)+r \omega^{2} ; \frac{\partial v}{\partial t}+2 \omega u=-\frac{1}{\rho r} \frac{\partial p}{\partial \theta}+\ddot{y} \sin (\omega t+\theta)-\ddot{z} \cos (\omega t+\theta),
$$

equation of continuity:

$$
\frac{\partial}{\partial r}(u r)+\frac{\partial v}{\partial \theta}=0
$$

and expressions for the forces acting on the system:

$$
\begin{aligned}
& M \ddot{z}+c_{z} z=\left.R h \int_{0}^{2 \pi} p\right|_{r=R} \sin (\omega t+\theta) d \theta ; \\
& M \ddot{y}+c_{y} y=\left.R h \int_{0}^{2 \pi} p\right|_{r=R} \cos (\omega t+\theta) d \theta,
\end{aligned}
$$

for limit conditions:

$$
\left.u\right|_{r=R}=0 ;\left.u\right|_{r=R_{1}}=\frac{\partial \zeta}{\partial t} ;\left.\quad p\right|_{r=R_{1}+\zeta}=0,
$$

where $p=p(r, \theta, t)$ is the liquid pressure at the point $\mathrm{A}(r$, $\theta), \rho$ is the density of the liquid; $h$ is height of cylindrical chamber; $\varsigma=\varsigma(r, \theta)$ is the radial deviation of the points of the free surface of the liquid.

Integration of the system of equations (1) - (3) is experiencing considerable difficulties. Therefore, in view of the study of the stability of the system, we turn to the analysis of its characteristic equation.

If we look for possible movements of the system in the form of principal oscillations, in which all generalized coordinates change with the same frequency (denote it $k$ ), setting the law of the change of the generalized rotor coordinates in the form:

$$
z=A_{1} \sin k t ; y=A_{2} \cos k t,
$$

and take into account only the waves of the $1^{\text {st }}$ order (since in the case of joint oscillations of the system a cylinder - a liquid in a liquid, waves arise only in the first order (the wavelength is equal to the length of the free surface of the liquid) [3]), we find the form of the inner surface of the liquid to the arbitrary constants $B_{1}$ and $B_{2}$ :

$$
\varsigma=B_{1} \cos [(k+\omega) t+\theta]+B_{2} \cos [(k-\omega) t-\theta] .
$$

We find the principal fluctuations of the fluid of the same frequency $k$.

The solution of the system of equations (1) - (3) with allowance for dependencies (4) and (5) is a system of four equations:

$$
\left.\begin{array}{c}
\frac{1}{2} A k^{2}+\left[\frac{2(k+\omega)^{2}}{\left(R / R_{1}\right)^{2}-1}+(3 \omega+k) \cdot(k+\omega)-\omega^{2}\right] B_{1}=0 ; \\
\frac{1}{2} B k^{2}+\left[-\frac{2(k-\omega)^{2}}{\left(R / R_{1}\right)^{2}-1}+(3 \omega-k) \cdot(k-\omega)+\omega^{2}\right] B_{2}=0 ; \\
-\left[k^{2}-\frac{c_{z}+c_{y}}{2(M+m)}\right] B+\frac{c_{z}-c_{y}}{2(M+m)} A+\frac{4 m(k-\omega)^{2}}{\left.\left(R / R_{1}\right)^{2}-1\right](M+m)} B_{2}=0 ; \\
\frac{c_{z}-c_{y}}{2(M+m)} B-\left[k^{2}-\frac{c_{z}+c_{y}}{2(M+m)}\right] A-\frac{4 m(k+\omega)^{2}}{\left.\mid\left(R / R_{1}\right)^{2}-1\right](M+m)} B_{1}=0,
\end{array}\right\}
$$

where: $m$ - mass of fluid in the rotor, $A=A_{1}-A_{2}, B=B_{1}-$ $B_{2}$. tion, its determinant must be zero. From this condition we obtain the equation of frequencies:

In order for system (6) to have a nontrivial solu- 


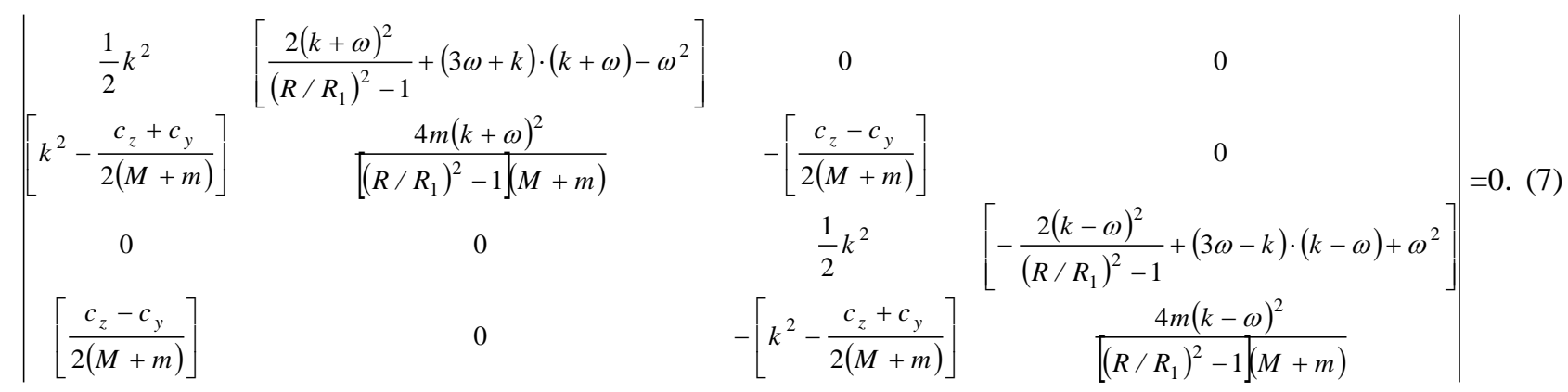

If $c_{z}=c_{y}=c$, then system (6) splits into two unbound systems, and the frequency equation (7) is to two independent expressions, since the left side of (7) for $c_{z}=c_{y}=c$ can be given as two multipliers, and the second one is obtained from the first substitute $k$ by $(-k)$. To solve the question of the stability of the vibrations of the system under consideration for $c_{z}=c_{y}=c$ it is enough to investigate one of the equations of the fourth degree, for example:

$$
\frac{2 m k^{2}(k-\omega)^{2}}{(M+m)\left(\left(R / R_{1}\right)^{2}-1\right]}+\left(k^{2}-\frac{c}{M+m}\right) \cdot\left[-\frac{2(k-\omega)^{2}}{\left(R / R_{1}\right)^{2}-1}+(3 \omega-k) \cdot(k-\omega)+\omega^{2}\right]=0 .
$$

The division of expression (7) into two independent equations means that for $c_{z}=c_{y}$ the principal oscillations of the system are circular. Equations (4) describe the motion of an axis of a cylinder in an elliptical trajectory. However, using the expressions $A=A_{1}-A_{2}, B=B_{1}-B_{2}$, an elliptic trajectory can be presented as a sum of two circular, with the angular velocities of these trajectories will be equal to $k$ and $(-k)$ :

$z=A_{1} \sin k t=\frac{A+B}{2} \sin k t=-\frac{A}{2} \sin (-k t)+\frac{B}{2} \sin k t$
$y=A_{2} \cos k t=-\frac{A-B}{2} \cos k t=-\frac{A}{2} \cos (-k t)+\frac{B}{2} \cos k t$,

which corresponds to the propagation of the wave in the forward and reverse directions (in a moving coordinate system a direct wave propagates towards the cylinder rotation, and the opposite is opposite to the rotation).

An indication of the instability of the system is the presence in the partial equation (8) of the roots with a negative imaginary part. Therefore, if among the four roots of equation (8) there is a couple of complex-conjugate, motion can be considered unstable.

The roots of equation (8) are the oscillation frequencies of the rotor-liquid system at $c_{z}=c_{y}=c$.

Taking the liquid stationary relative to the rotor ( $\left.A_{1}=A_{2}, B_{1}=B_{2}=0\right)$, we obtain the partial frequencies of the propagation of direct and reverse waves and the frequency of oscillations of the rotor-liquid system:

$$
\begin{aligned}
& k_{\text {liq }}^{+}=\omega \cdot b=\frac{\omega}{1-\sqrt{\frac{1-\left(R_{1} / R\right)^{2}}{2}}} ; \\
& k_{\text {liq }}^{-}=\omega \cdot a=\frac{\omega}{1+\sqrt{\frac{1-\left(R_{1} / R\right)^{2}}{2}}} .
\end{aligned}
$$

We express (8) through the partial frequencies:

$$
\alpha k^{2}(k-\omega)^{2}-\left(k^{2}-k_{p}^{2}\right) \cdot\left(k-k_{l i q}^{+}\right) \cdot\left(k-k_{l i q}^{-}\right)=0,
$$

where: $\alpha=\frac{2 m}{M+m} \cdot \frac{1}{1+\left(R / R_{1}\right)^{2}}<1 ; k_{p}^{2}=\frac{c}{M+m}-$ the partial frequency of the rotor with the liquid in it is in a state of rest.

It is shown in [12] that for any $\alpha, a, b, k_{p}^{2}$, which is the constructive parameters of a system, there is always a rotational speed of the rotor $\omega$, in which the equation (8) will have complex roots, that is, the system will be unstable. For the specific parameters of the system by numerical methods, equating the discriminant equation (9) to zero or using the Euclidean algorithm, one can determine the boundaries of the area of instability.

Thus, the rotating rotor is partially filled with liquid and installed on elastic supports, at some speeds of rotation, it loses its stability. These speeds are not discrete. When $c_{z}=c_{y}$ there is one zone of instability, located around the rotational speed $\omega_{c r}$, at which there is a coincidence of the partial frequency of the rotor $k_{p}$ with a partial velocity of propagation of the return wave in the liquid $\left(k_{\text {liq }}^{-}=\omega \cdot a\right)$.

With this condition $\left(k_{\text {liq }}^{-}=\omega \cdot a\right)$ we get the formula of critical velocity:

$$
\omega_{c r}=\sqrt{\frac{c}{M+m}} \cdot\left[1+\sqrt{\frac{1-\left(R_{1} / R\right)^{2}}{2}}\right]
$$

Expression (10) defines a central point of an unstable zone. The main parameter characterizing the width of an unstable area is $\alpha$.

\section{Numerical example}

The parameter $\alpha$ is a constructive parameter of the rotor-fluid system, which depends on the degree of filling of the $\mathrm{ABD}$ chamber with a liquid. In addition, it was numerically checked that an increase in the mass of the fluid leads to the expansion of the instability area. So for such 
parameters of the system: the weight of the rotor with the camera $\operatorname{ABD}(M)-7.3 \mathrm{~kg}$, the weight of the imbalance $\left(m_{d}\right)-0,1 \mathrm{~kg}$, the external radius of the camera $\mathrm{ABD}(R)-$ $0.2 \mathrm{~m}$, the height of the chamber $\operatorname{ABD}(h)-0.05(m)$, mass of liquid $(m)-0.05-0.45 \mathrm{~kg}$, liquid density $(\rho)-$ $1000 \mathrm{~kg} / \mathrm{m}^{3}$, liquid temperature $(t)-18^{\circ} \mathrm{C}$, critical speed of the rotor $\left(\omega_{c r}\right)-9.64-14.00 \mathrm{~Hz}$, the relationship between the degree of filling of the chamber $\mathrm{ABD}$ and the coefficient $\alpha$ has the form shown in Fig. 4.

Width of the unstable area, $\mathrm{Hz}$

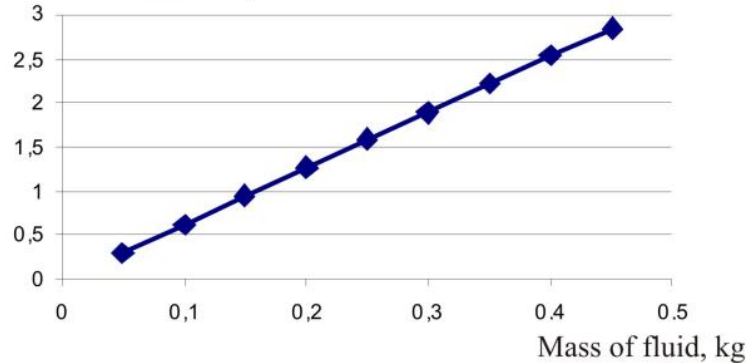

Fig. 4 Increase of the width of the area of motion instability rotor system - ABD - liquid depending on the filling of the ABD chamber with liquid

Thus, when the chamber is filled with a liquid weighing $50 \mathrm{~g}$, the instability area of the system is $10.726-11.036 \mathrm{~Hz}$ (the width of the instability area is $0.31 \mathrm{~Hz}$ ), and when the $450 \mathrm{~g}$ of liquid is filled, the system instability area is $10.047-12.885 \mathrm{~Hz}$ (the width of the instability area is $2.839 \mathrm{~Hz}$ ).

\section{Conclusions}

Effect of the volume of liquid on the efficiency of the system balancing on the resonant rotor frequencies was determined.

A numerical model of auto-balancing device (ABD) was created. The presence of the areas of instability of the rotor-fluid system and the dependence of the area width on the mass of the fluid filling the chamber of the ABD can be explained by an increase in the amplitudes of the oscillations of the system when the ABD chamber is filled with more liquid than necessary for balancing. The wave frequency in the fluid resonates with the rotor's rotational speed.

\section{References}

1. Nikiforov, A. 2013. Condition of the problem of equilibration of rotors, Bulletin of Scientific and Technological Development 4 (68): 20-28 (in Russian).

http://www.vntr.ru/nomera/2013-468/.

2. Seok-Ho Son; Sang-Bin Lee; Dong-Hoon Choi. 2012. Experiment-based design optimization of a washing machine liquid balancer for vibration reduction, International Journal of Precision Engineering and Manufacturing, August 2012, 13(8): 1433-1438. https://doi.org/10.1007/s12541-012-0188-y.

3. Kasahara, M.; Kaneko, S.; Oshita, K.; Ishii, H. 2000. Experiments of liquid motion in a whirling ring, Proceedings of the Dynamics and Design Conference 2000, 5-8 August 2000, Japan Society of Mechanical Engineers, Tokyo, Japan, pp.1-6.

4. Nakamura, T. 2009. Study on the improvement of the fluid balancer of washing machines. In Proceedings of the 13th Asia-Pacific Vibrations Conference, 22-25 November 2009, University of Canterbury, New Zealand, p. 1-8.

5. Bae, S.; Lee, J. M.; Kang, Y. J.; Kang, J. S.; Yun, J. R. 2002. Dynamic analysis of an automatic washing machine with a hydraulic balancer. J. Sound Vib. 257: 3-18. https://doi.org/10.1006/jsvi.2001.4162.

6. Jung, C.-H.; Kim, C.-S.; Choi, Y.-H. 2008. A dynamic model and numerical study on the liquid balancer used in an automatic washing machine, J. Mech. Sci. Tech. 22: 1843-1852. https://doi.org/10.1007/s12206-008-0623-2.

7. Majewski T. 2010. Fluid balancer for a washing machine. In Proceedings of the XVI International Congress, SOMIM (Society of Mechanical Engineers of Mexico), p. 1-10. somim.org.mx/articulos2010/memorias/.../A4/A4_29.pdf.

https://doi.org/110.4028/www.scientific.net/SSP.147149.374.

8. Chen, H.-W.; Zhang, Q.; Fan, S.-Y. 2011. Study on steady-state response of a vertical axis automatic washing machine with a hydraulic balancer using a new approach and a method for getting a smaller deflection angle, J. Sound Vib. 330: 2017-2030.

https://doi.org/10.1016/j.jsv.2010.11.006.

9. Urbiola-Soto, L.; Lopez-Parra, M. 2011. Dynamic performance of the Leblanc balancer for automatic washing machines, J. Vibr. Acoust. 133: 041014-1041014-8. https://doi.org/110.1115/1.4003597.

10. Royzman, V.; Bubulis, A.; Drach, I. 2009. System Analysis of Automatic Balancing (Self-Balancing) Machine Rotors with Liquid Working Bodies, Solid State Phenomena 147-149: 374-379. https://doi.org/110.4028/www.scientific.net/SSP.147149.374 .

11. Stewartson, K., J. 1959. Fluid Mech. 5: 577-592. [Russian translation: The Book Mechanics 6 (64):3-19, 1960.

12. Marhashov, L. 1962. Oscillations and stability of a rigid shell with an ideal fluid on elastic supports (to the theory of a carousel waterchanel, PMTF 6: 32-39. (in Russian).

13. Cunico, M.W.M. 2015. Characterization and Modelling of LeBlanc Hydrodynamic Stabilizer, A Novel Approach for Steady and Transient State Models, Modelling and Simulation in Engineering 2015. http://dx.doi.org/10.1155/2015/729582.

14. Royzman, V., Drach, I. 2005. Improving theory for automatic balancing of rotating rotors with liquid self balancers. Mechanika 4(54): 38-44. http://mechanika.ktu.lt/index.php/Mech/article/view/14 500. 
I. Drach, A. Bubulis, D. Mažeika,

R. Kandrotaite Janutienè, D. Juodvalkis

INVESTIGATION OF SMALL MOTIONS OF LIQUID

IN CYLINDRICAL CHAMBER

OF AUTO-BALANCING DEVICE

\section{S u m m a r y}

The article deals with the process of wave forming on the surface of a liquid in a chamber of autobalancing device partially filled with a liquid for rotors with a vertical axis of rotation under non-stationary modes of the system's motion. In non-stationary processes, the possible wave formation causes a dynamic instability in the operation of the machine and the increase of vibrations under certain operating modes. In this article the case when the rotor is installed in elastic supports is solved, the joint movements of the rotor - liquid system are considered and their stability is investigated.

Keywords: rotor, imbalance, automatic balancing (selfbalancing), auto-balancing device (ABD), process of wave forming on the surface of a liquid.

Received March 21, 2018 Accepted April 18, 2018 